\title{
APRENDIZAGEM ORGANIZACIONAL E CAPACIDADE ABSORTIVA: UMA ANÁLISE TEÓRICA NO CONTEXTO DA GESTÃO DO CONHECIMENTO
}

\section{Resumo}

$\mathrm{Na}$ sociedade da informação, a globalização e a hipercompetitividade de mercado forçaram as empresas a se reinventarem e,com isso,construírem inovações constantes e sistematizadas com o intuito de alcançarem vantagens competitivas sustentáveis. Diferenciam-se as organizações que tiverem maior capacidade para construir aprendizagem organizacional e absorver conhecimentos, gerando valor para seus negócios e se reposicionando em seus mercados com o passar dos anos. Nesse contexto, a gestão do conhecimento passou a ser fator chave nos negócios, possibilitando mais agilidade nas tomadas de decisões, além de gerar ciclos de inovações e melhorias contínuas e contribuir para que as organizações conquistassem lugar de destaque no mundo competitivo. Neste sentido, este estudo teve como questão de pesquisa: qual a relação da aprendizagem organizacional e da capacidade absortiva no contexto da gestão do conhecimento nas organizações da sociedade da informação? No intuito de buscar respostas para esta questão de pesquisa, este estudo teve por objetivo analisar a relação da aprendizagem organizacional com a capacidade absortiva nas organizações, demonstrando sua relação com a gestão do conhecimento. Para tanto, foi realizada uma pesquisa teórica de fins exploratóriodescritivo por meio de levantamento bibliográfico. Os elementos teóricos levantados confirmaram a relevância desta pesquisa, identificaram que a o conhecimento é insumo da capacidade absortiva e da aprendizagem organizacional, evidenciando que a gestão do conhecimento desempenha papel chave na integração entre estas duas temáticas e que, para amplificar sua análise, a abordagem interdisciplinar dos temas é imprescindível.

Palavras-chave: Aprendizagem organizacional. Capacidade absortiva. Gestão do conhecimento. Interdisciplinaridade.

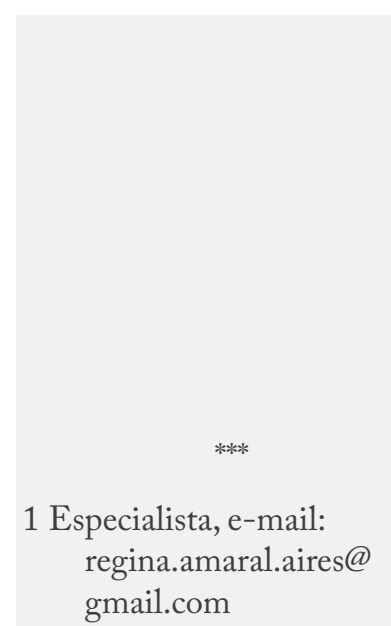

$* * *$ 


\section{INTRODUÇÃO}

De acordo com Davenport e Prusak (1998, p. 16), “[...] cada vez mais as atividades baseadas no conhecimento e voltadas para o desenvolvimento de produtos e processos estão tornando as principais funções internas das empresas e aquelas com maior potencial de obtenção de vantagem competitiva”. Estas são as características que identificam as organizações da sociedade do conhecimento, também denominada de sociedade da informação ou economia do conhecimento.

O conhecimento “[...] é conteúdo ou processo efetivado por agentes humanos ou artificias em atividades de geração de valor científico, tecnológico, econômico, social ou cultural." (UFSC, 2016).

A sociedade da informação se caracteriza por um mercado instável, de rápidas mudanças, em que as organizações são desafiadas diariamente a manteremse vivas (DAVENPORT; PRUSAK, 1998; TEIXEIRA FILHO, 2000).

Neste cenário, a capacidade de absorver conhecimentos em um ciclo de aprendizagem contínuo passa a ser fator crítico de sucesso, ou seja, as organizações que melhor gerirem seus conhecimentos terão mais chances de conquistarem vantagens competitivas sustentáveis e se manterem na dianteira de seus negócios (COHEN; LEVINTHAL, 1990; ZAHRA; GEORGE, 2002).

A capacidade absortiva é definida como a potencialidade de uma organização em observar no ambiente externo novas informações de alto valor para seus negócios, assimilá-las, aplicá-las e de reconfigurar sua operação, inovando de forma continuada (COHEN; LEVINTHAL, 1990; ZAHRA; GEORGE, 2002).

Para Lane; Koka; Pathak (2006), a capacidade de absorção é um elemento essencial para o sucesso de qualquer organização, pois reforça, complementa e transforma os atuais conhecimentos, possibilitando a construção de novos saberes por meio da aprendizagem.

Tal aprendizagem pode ser compreendida como um processo de mudança do pensamento individual para o compartilhado, ou seja, seu foco está no processo e não no resultado. Esta aprendizagem, também denominada de aprendizagem organizacional, busca investigar como as organizações aprendem nos diferentes níveis (indivíduo, grupo,organização e interorganização) (EASTERBY-SMITH; LYLES, 2005).

Fiol e Lyles (1985) enfatizaram que aprendizagem organizacional tem um íntimo elo com a capacidade de mudança, adaptação e ajuste da organização como forma de responder às situações do ambiente em que a organização está inserida. Corroborando, Alves, Steil e Santos (2015, p. 3) apontam que diversos autores (EASTERBY-SMITH; LYLES, 2005; ARGOTE; MIRON-SPEKTOR, 2011; CROSSAN; LANE; WHITE, 1999; CROSSAN; MAURER; WHITE, 2011; WILSON; GOODMAN; CRONIN, 2007) afirmaram que a "[...] aprendizagem organizacional é o processo de mudança na cognição e no comportamento."

Neste contexto, surge a questão de pesquisa deste estudo: qual a relação da aprendizagem organizacional e a capacidade absortiva no 
contexto da gestão do conhecimento nas organizações da sociedade da informação? No intuito de buscar respostas para esta questão de pesquisa, este estudo teve por objetivo analisar a relação da aprendizagem organizacional com a capacidade absortiva nas organizações, demonstrando sua relação com a gestão do conhecimento nas organizações da sociedade da informação que buscam a construção de vantagens competitivas sustentáveis.

Este estudo classifica-se como uma pesquisa teórica, com objetivos exploratório descritiva, realizada por meio de uma revisão bibliográfica (FREIRE,2013), a partir de artigos científicos e livros de autores referência dos temas.

Desta forma, com base nas bibliografias levantadas, é possível identificar a relação dos temas abordados neste estudo, aprendizagem organizacional e capacidade absortiva, sendo o conhecimento o eixo central destes dois temas.

Conforme apresentaremos nas próximas seções, as abordagens dos temas desta pesquisa são correlacionados e interdisciplinares. "A interdisciplinaridade pode ser entendida como uma condição fundamental do ensino e da pesquisa [...] um ponto de cruzamento entre atividades (disciplinares e interdisciplinares) com lógicas diferentes" (LEIS, 2005, p. 3). Para Leis (2005, p. 9), “[...] o que interessa é o avanço do conhecimento através de suas diferentes manifestações".

$\mathrm{Na}$ próxima seção, será aprofundado o relacionamento destes temas com a gestão do conhecimento.

\section{CAPACIDADE ABSORTIVA, APRENDIZAGEM ORGANIZACIONAL E
GESTÃO DO CONHECIMENTO}

O conhecimento é o aspecto central da capacidade de absorção e da aprendizagem organizacional e não é absorvido de forma igual nas diferentes organizações, ou seja, sua internalização acontece de forma diferente em cada uma, de acordo com as experiências, os perfis das pessoas de suas equipes e a cultura da empresa (HOTHO; BECKERRITTERSPACH; SAKA-HELMHOUT, 2012). Esta diferenciação está relacionada à forma como as informações são compartilhadas no ambiente organizacional. Dependerá, também, da cultura organizacional de cada instituição, ou seja, da forma como acontece a interação entre as pessoas e entre os grupos de cada empresa (ZAHRA; GEORGE, 2002).

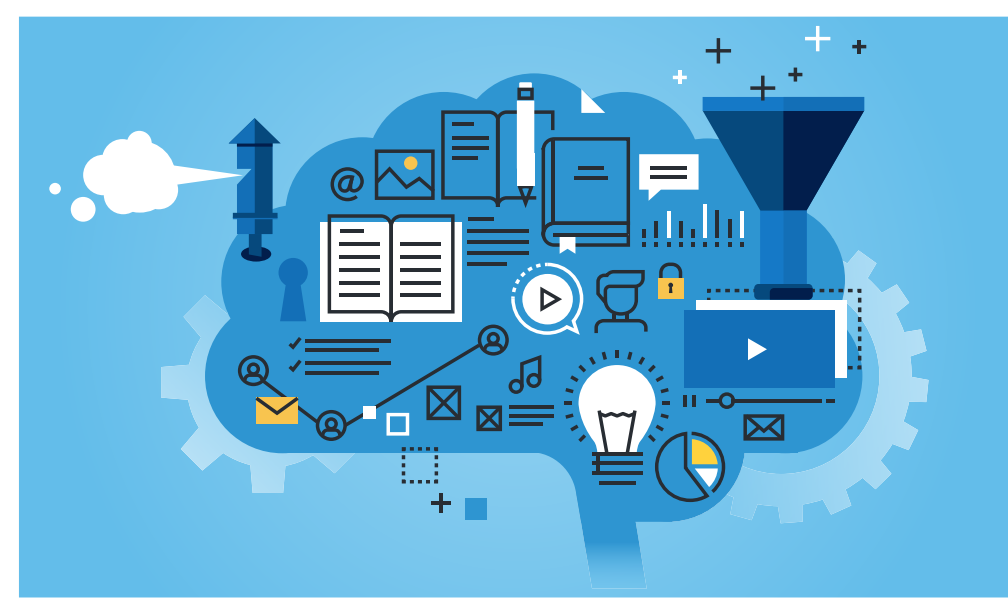


Cohen e Levinthal (1990) destacaram

que a capacidade absortiva de uma organização, também definida como capacidade de absorção, não depende apenas de seu relacionamento com o ambiente externo, mas principalmente do compartilhamento de conhecimento dentro da instituição.

Inter-relaciona-se, portanto, aprendizagem organizacional com a capacidade de seus indivíduos e grupos de utilizar o que já sabem para assimilar novos conhecimentos e transformar as suas práticas, ou seja, absorver conhecimentos.

O estado da arte do processo de capacidade de absorção leva à plena aprendizagem organizacional, em que as falhas em um impactam o outro, demonstrando a recursividade, que é mediada por mecanismos de integração na organização (CAMISÓN; FORÉS, 2011).Na mesma linha,Lane e Lubatkin (1998) afirmam que a relação existente entre a capacidade absortiva e a de aprendizagem organizacional é de recursividade, pois, ao passo que uma organização desenvolve seu aprendizado, aumenta sua capacidade de compreender e desenvolver novos conhecimentos, expandindo seus saberes e ampliando sua aprendizagem.

Versiani e Fischer (2008) destacam a relação da aprendizagem organizacional com a capacidade absortiva das organizações, afirmando que, para existir o aprendizado organizacional,é necessário desenvolvimento de conhecimentos, processo que deve acontecer de forma fluida. Este fluxo de conhecimento é elemento chave tanto da aprendizagem organizacional quanto da capacidade de absorção, ou seja, há uma interface entre os conceitos.
Para Picoli E TAKaHASHI

(2015), A APRENDIZAGEM

ORGANIZACIONAL ABARCA

ASPECTOS COMO MUDANÇAS,

HÁBITOS, EXPERIÊNCIAS,

FATORES COMPORTAMENTAIS

E COGNITIVOS, ENTRE

OUTROS, QUE A TORNAM MAIS

AMPLA E A DIFERENCIAM

DA CONCEITUAÇÃO DE

CAPACIDADE ABSORTIVA,

OU SEJA, NÃO SE TRATA DA

MESMA COISA, PORÉM, SÃO

TERMOS QUE SE RELACIONAM

DE MODO RECURSIVO E

ATUAM COM A MESMA BASE, O CONHECIMENTO.

Deste modo, a capacidade absortiva pode envolver um processo de aprendizagem em diversos níveis, não necessariamente no nível organizacional. Para que a aprendizagem seja construída em nível organizacional,é necessário que mudanças profundas ocorram, reverberando, de alguma forma, os valores institucionais.

Em uma pesquisa realizada por Kurtz, Santos e Steil (2013), os autores apresentam estudos que tratam de capacidade absortiva comumente relacionada à aprendizagem organizacional e à gestão do conhecimento, sinalizando que a capacidade absortiva deve ser considerada como um tipo específico de aprendizagem que diz respeito à relação da organização com os conhecimentos externos. Os autores destacaram o estudo de Sun e Anderson (2010), An examination of the relationship between absorptive capacity and organizational learning, and a proposed integration, que aborda a relação da 
capacidade absortiva com aprendizagem organizacional. No Brasil,Picoli e Takahashi (2015) também apresentam em seu estudo intitulado "Capacidade de Absorção, Aprendizagem Organizacional e Mecanismos de Integração Social" a relação destas temáticas.

Em contextos turbulentos, de alta competitividade, como é o da sociedade da informação, estudos têm demonstrado que a capacidade absortiva influencia a aprendizagem organizacional e a inovação de forma positiva (GARCÍA-MORALES; RUIZ-MORENO; LLORENS-MONTES, 2007).

Neste sentido, Nonaka e Takeuchi (1997) constataram há 19 anos o que Crossan e Apaydin (2010) disseram em 2010, que há uma relação próxima entre aprendizagem organizacional e inovação. Para os autores, a aprendizagem organizacional é um fator determinante para a inovação, associando à relação dos temas com a gestão do conhecimento e com o fomento da competividade organizacional.

Para Teixeira Filho (2000, p. 22), “A gestão do conhecimento pode ser vista como uma coleção de processos que governa a criação, disseminação e utilização do conhecimento para atingir plenamente os objetivos da organização.”

Desde a década de 90, a gestão do conhecimento passou a ser estratégica para qualquer organização que pretende manter-se no mercado e consolidar vantagem competitiva sustentável. "Junto com essa realidade vem a constatação de que a inovação permanente é um fator crítico de sucesso das organizações.” (TEIXEIRA FILHO, 2000, p. 42). O condutor central da inovação,segundo a OCDE (2005), éa gestão de conhecimento. Na mesma linha, Ueno, Santos e Dandolini (2016, p. 1) apresentam que “[...] as oportunidades baseadas em conhecimento são essenciais para o processo de inteligência competitiva, induzindo e fortalecendo a capacidade inovativa das empresas."

A dinâmica do mundo competitivo atual exige respostas rápidas das organizações.

Destacam-se aquelas que conseguem com mais agilidade e maestria gerir seu conhecimento.

Neste sentido, Teixeira Filho (2000) e Davenport e Prusak (1998) defendem que a verdadeira vantagem competitiva está na capacidade e na velocidade do aprendizado das pessoas nas organizações e que o conhecimento é fundamental para o sucesso e a sobrevivência das organizações.

\section{PARA LANE E LUBATKIN \\ (1998), É PRECISO ENTENDER \\ COMO OCORRE O PROCESSO \\ DE APRENDIZADO E ABSORÇÃO \\ DO CONHECIMENTO PELAS \\ PESSOAS, POIS É A MANEIRA \\ COMO OS MEMBROS DA \\ ORGANIZAÇÃO APRENDEM, \\ COMBINAM E APLICAM O \\ CONHECIMENTO QUE GERA \\ VANTAGEM COMPETITIVA.}

$\mathrm{Na}$ sociedade da informação, o conhecimento destaca-se como fator de competividade de mercado.As dinâmicas do capitalismo desafiam as organizações a se reinventarem para garantir sua perenidade e competitividade. Deste modo, investir em gestão do conhecimento passa a ser estratégico (DA SILVA, 2004). "O 
conhecimento tornou-se o fator econômico mais importante no ambiente competitivo das organizações [...] é um fator decisivo para a sobrevivência da empresa no novo ambiente competitivo." (TEIXEIRA FILHO, 2000, p. $17-21)$.

Picoli e Takahashi (2015) apresentam que os mecanismos de integração social fazem o elo entre aprendizagem organizacional e a capacidade absortiva, e que as distintas integrações sociais desenvolvidas nas organizações contribuem para que cada uma chegue a resultados diferentes.A criação de valor, a partir do conhecimento, está relacionada com a capacidade de absorção das organizações em gerir da melhor forma os conhecimentos novos e externos gerados em seu processo de aprendizagem (JANSEN; VAN DEN BOSCH; VOLBERDA, 2005).

Neste sentido, Davenport e Prusak (1998, p. 14-16) destacam que "Novo é reconhecer o conhecimento como um ativo corporativo e entender a necessidade de geri-lo e cercá-lo do mesmo cuidado dedicado à obtenção de valor de outros ativos mais tangíveis. A necessidade de extrair o máximo de valor do conhecimento organizacional é maior agora que no passado.”

Portanto, pode-se verificar na literatura abordada a relação dos temas capacidade absortiva, aprendizagem organizacional e sua relação com a gestão do conhecimento. Na próxima seção, será aprofundada a relação destes temas com o conhecimento sob o ponto de vista de fator gerador de valor.

\section{CONHECIMENTO: ATIVO GERADOR DE VALOR}

A Indústria 4.0, também denominada de Manufatura Avançada (CNI, 2016) e Quarta Revolução Industrial (LAURETH, 2014), “[...] caracteriza-se por uma fábrica inteligente de alta complexidade tecnológica em que as máquinas, os produtos, os insumos e clientes estão conectados pela comunicação de dados para monitoramento e tomada de decisão." (LAURETH, 2014). Nesta nova Revolução Industrial, o fator chave será a gestão do conhecimento aliado à convergência tecnológica, buscando a geração de valor aos negócios das organizações para a construção de vantagens competitivas sustentáveis (CNI, 2016). Notase que o conhecimento é o ativo central desta nova era de produção.
Teixeira Filho (2000) e Davenport e Prusak (1998) afirmam que, em uma economia globalizada, altamente competitiva, com ciclos de criação de produtos cada vez mais curtos, as organizações acabam se diferenciando pelo que elas sabem e pela forma como conseguem usar este conhecimento, ou seja, o conhecimento passa a ser a maior vantagem competitiva sustentável, sendo um capital que precisa ser gerenciado.

Nesta linha, Serrat (2008, p. 1) apresenta que o conhecimento é um ativo organizacional que só tem valor se entregue no momento certo, disponível no lugar certo, de forma direta, com o menor custo possível e com a qualidade 
exigida.É imaterial, difícil de medir e pode ser usado por vários processos ao mesmo tempo. Já a gestão do conhecimento "[...] é obter o conhecimento certo paras as pessoas certas no momento certo, e ajudá-los (com incentivos) para aplicá-la de maneira que se esforçam para melhorar o desempenho organizacional.”

Para Nonaka e Takeuchi (1997), a criação do conhecimento organizacional é a chave para a inovação nos negócios. Esta é a base das empresas japonesas, pois elas são peritas em fomentar a inovação de forma contínua, incremental e em espiral.Esses são os ingredientes para a obtenção de vantagens competitivas sustentáveis.

Háquatroformasdetransformaroconhecimento tácito em explícito: pela socialização, externalização, combinação e internalização. Para que esta espiral do conhecimento aconteça e gere valor e vantagens competitivas sustentáveis, é necessária a integração entre os processos empresariais e as estratégias da organização. Para isto, é imprescindível a integração das pessoas, das redes de informação e o emprego da tecnologia da informação, ou seja, precisa acontecer o processo de aprendizagem organizacional e a absorção dos conhecimentos (NONAKA; TAKEUCHI, 1997).

Não há maneira de manter o segredo de produção por muito tempo. As vantagens de novos produtos são sustentáveis por um curto espaço de tempo, “[...] porém, a empresa rica em conhecimento e gestora do conhecimento terá passado para um novo nível de qualidade, criatividade ou eficiência. A vantagem do conhecimento é sustentável porque gera retornos crescentes e dianteiras continuadas." (DAVENPORT; PRUSAK, 1998, p. 20).

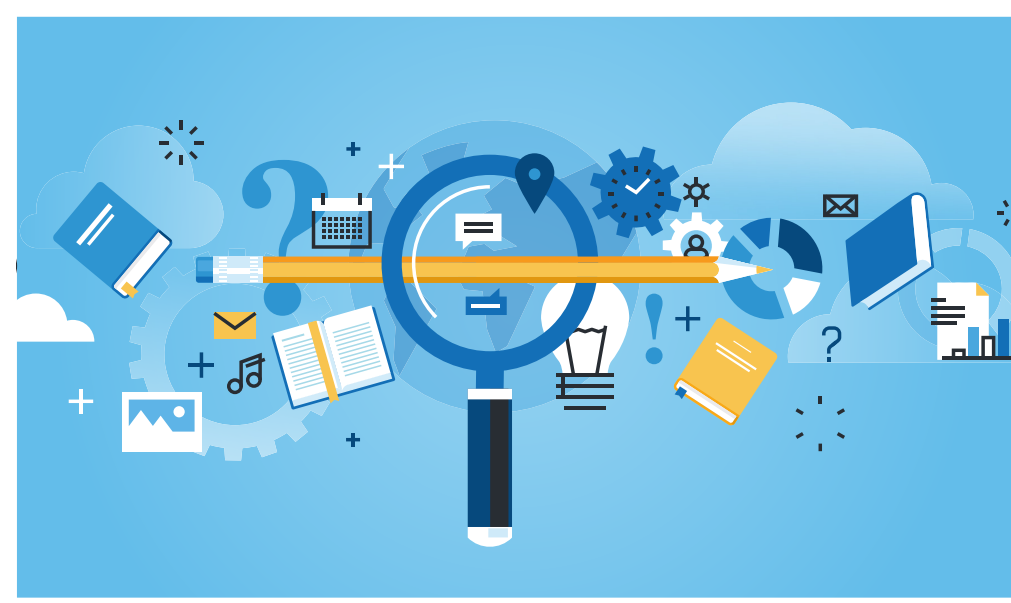

Neste contexto, Dávila (2016) apresenta que uma considerável quantidade de pesquisas tem sido publicada em diversas áreas sobre o impacto da capacidade absortiva das organizações como conversor de conhecimento em valor, favorecendo o desempenho organizacional.

Analisando o apresentado nas seções anteriores, percebe-se a aderência e a inter-relação dos conceitos aprendizagem organizacional e capacidade absortiva com o conceito de conhecimento como agregador de valor nos resultados organizacionais (UFSC, 2016).

Na próxima seção, será abordada a importância de um olhar interdisciplinar para aprofundar o debate destes temas. 


\section{O PAPEL DA INTERDISCIPLINARIDADE NO CONTEXTO}

Conforme apontado pelos autores mencionados nas seções 2 e 3 desta pesquisa, o conhecimento é um bem que precisa ser gerenciado, sendo um capital que pode gerar vantagens competitivas sustentáveis para os negócios das organizações. Sendo o conhecimento o insumo, tanto para a aprendizagem organizacional como para a capacidade abortiva das instituições, são capazes de gerar valor para a organização.

De acordo com a publicação da Confederação Nacional da Indústria (CNI, 2016) "Desafios para a Indústria 4.0 no Brasil”,

As novas formas de produção
decorrentes da Indústria 4.0 exigem
profissionais com formação distinta
das existentes. A integração de
diversas formas de conhecimento,
característica desse modo de produção,
exigirá equipes multidisciplinares,
com elevado nível de conhecimento
técnico e com capacidade de interação
de diferentes áreas de conhecimento
(p. 29).

No contexto da Indústria 4.0, Laureth (2014) aponta que a convergência tecnológica, criará sinergia entre campos científicos e tecnológicos desta era da manufatura avançada, promovendo uma articulação interdisciplinar dos conhecimentos cognitivo e técnico. O desenvolvimento de pesquisas que inter-relacionam diferentes campos das ciências passam a ser bastante relevantes.

Quanto ao constructo da capacidade absortiva, de acordo com Santos (2013), o tema em si já pode ser considerado interdisciplinar, pois tem origem na economia e aolongo dos anos tem sido estudada por outros campos do conhecimento, como teoria das organizações, aprendizagem organizacional, inovação, alianças estratégicas, sistemas de informação. Assim, estudar a capacidade de absorção poderá fornecer insumos para a gestão dos conhecimentos externos das organizações relacionados à aprendizagem organizacional.

\section{AprendizAGEM}

ORGANIZACIONAL E

CAPACIDADE ABSORTIVA

SÃO TEMAS QUE ROMPEM

AS FRONTEIRAS DE UMA

DISCIPLINA, OU SEJA, É

INTERDISCIPLINAR (AUSBURG,

2006; FRIGOTTO,

2008; POMBO, 2005;

LEIS, 2005), POIS SÃO

CONCEITOS DISCUTIDOS NA

ADMINISTRAÇÃO, ECONOMIA,

GESTÃO DO CONHECIMENTO,

ENTRE OUTRAS (DÁVILA,

2016).

A abordagem interdisciplinar dos temas deste estudo, capacidade abortiva e aprendizagem organizacional, sob a luz da gestão do conhecimento, são prioritárias para avançar a um patamar superior de discussão. Neste sentido, a interdisciplinaridade permite cruzar ideias e métodos provenientes de distintas disciplinas, para produzir algo novo e útil em uma intensidade que não poderia ser alcançada utilizando-se conceitos e métodos de uma única disciplina (CHENG et al.; 2009). Corroborando, Freire 
(2012, p. 47) apresenta que a interdisciplinaridade é importante "[...] pela necessidade de buscar múltiplos olhares sobre o objeto de estudo para poder entendê-1o", ou seja, “[...] buscar apoio teórico em diferentes ciências, como a administração, a pedagogia, a psicologia e, a engenharia e gestão do conhecimento".
Após destacar a importância da característica interdisciplinar na abordagem dos temas deste estudo, serão apresentadas, na próxima seção, as considerações finais e os desafios da pesquisa destas temáticas.

\section{DESAFIOS}

A aprendizagem organizacional e a capacidade absortiva foram, originalmente, relacionadas no artigo clássico de Cohen e Levinthal (1990), intitulado Absorptive capacity: a new perspective on learning and innovation, dando origem a algumas pesquisas que tratam destas temáticas, dentre as quais, destaca-se ao estudo de Zahra e George(2002),intitulado: "Absorptive capacity: A review, reconceptualization, and extension" (KURTZ; SANTOS; STEIL, 2013).

Picoli eTakahashi (2015) destacam que os estudos realizados acerca destas temáticas deflagraram reflexões e apontaram que a internalização de um novo conhecimento ocorre de maneira fluida e processual, sinalizando a existência de interface entre a capacidade de absorção e a aprendizagem organizacional.

Zangiski, Lima e Costa (2009) deixam claro que a aprendizagem organizacional surge como importante processo para a formação, manutenção e desenvolvimento de competências e capacidades para a competitividade das organizações.

No estudo "Vencendo os desafios do crescimento: o método 'aprender a crescer' para pequenas e médias empresas brasileiras", no qual são abordadas a capacidade absortiva e a aprendizagem organizacional de pequenas e médias empresas brasileiras, North; Silva Neto; Dávila (2013) identificaram os seguintes desafios enfrentados por organizações de pequeno e médio porte em busca da competitividade: carga tributária, falta de pessoal qualificado, ausência de unidades internas com foco em inovação, insuficiência de recursos financeiros, entre outros. Para enfrentar estes desafios, Dávila (2016) sugeriu, no estudo "Relações entre práticas de gestão do conhecimento, capacidade absortiva e desempenho: evidências do sul do Brasil”, publicado três anos mais tarde, que o desenvolvimento de capacidades absortivas são chaves, pois, para obter vantagens competitivas sustentáveis,é preciso ir além da busca pelos resultados, isto é, é preciso desenvolver competências que possibilitem a combinação e recombinação de conhecimentos para garantir a competitividade no ambiente da sociedade da informação, com características distintas e dinâmicas.

Picoli e Takahashi (2015) apresentam, no estudo "Capacidade de Absorção, Aprendizagem Organizacional e Mecanismos de Integração Social", que os mecanismos de integração social desempenham papel chave para fomentar a aprendizagem e, consequentemente, aumentar a capacidade de absortiva. Uma vez que a pesquisa foi realizada em um universo limitado, instituições de ensino públicas do Estado do Paraná, os autores sugeriram que novas pesquisas nessa temática fossem realizadas em outros ambientes 
organizacionais, expandindo o número de estudos empíricos que possibilitem uma melhor compreensão da relação de recursividade das temáticas abordadas no estudo. Destacam que, nestes estudos futuros, seria importante delinear os principais elementos que podem configurar os mecanismos de integração social, buscando identificar o impacto gerado no fluxo de aprendizagem e na absorção do conhecimento.

Angeloni e Steil(2011) observam uma carência de estudos que exploram o alinhamento dos temas aprendizagem e conhecimento, também abordados nesta redação, destacando esta área promissora para pesquisas futuras. Sugerem, ainda, a realização de estudos empíricos que abordem estas temáticas relacionadas a estratégias de negócios.

Embora existam diversas pesquisas relacionadas aos temas propostos para esta pesquisa, capacidade absortiva e aprendizagem organizacional, com base nas leituras realizadas, pode-se perceber que há uma lacuna a ser explorada: uma análise detalhada sobre as publicações que relacionam estes dois conceitos. Assim, sugere-se, para pesquisas futuras, o desenvolvimento de uma análise bibliográfica para investigação em profundidade da relação entre estes dois temas sob o olhar da gestão do conhecimento, identificando o que o mundo científico tem abordado sobre este tema.

Por fim, alinhado ao objetivo de analisar a relação da aprendizagem organizacional com a capacidade absortiva nas organizações, demonstrando sua relação com a gestão do conhecimento,além de responder à questão de pesquisa deste estudo: qual a relação da aprendizagem organizacional e a capacidade absortiva no contexto da gestão do conhecimento nas organizações da sociedade da informação?, foram levantados elementos teóricos que confirmaram a relevância desta pesquisa, conforme apresentado nas seções anteriores deste artigo.

\section{Foi identificado que o conhecimento} é insumo da capacidade absortiva e da aprendizagem organizacional, evidenciando que a gestão do conhecimento desempenha papel chave na integração entre estas duas temáticas e que, para amplificar sua análise, a abordagem interdisciplinar dos temas é imprescindível. 


\section{ORGANIZATIONAL}

LEARNING AND

ABSORPTIVE

CAPACITY: A

THEORETICAL

ANALYSIS IN CONTEXT

OF KNOWLEDGE

MANAGEMENT

\begin{abstract}
In the information society, globalization and market bypercompetitiveness haveforced companiestoreinvent themselves, therebybuilding constantandsystematized innovations with the aim of achieving sustainable competitive advantages. Organizations with greater capacity to build organizational learning and absorb knowledge, generating value for their businesses and repositioning themselves in their markets over the years are differentiated. In this context, knowledge management has become a key factor in business, allowing more agile decision making, generating cycles of innovations and continuous improvements, which contribute to organizations gaining a prominent place in the competitive world. In this sense, this study had as a research question: what is the relationship between organizational learning and the absorptive capacity in the context of knowledge management in organizations of the information society? In order to find answers to that research question, thisstudyaimed to analyze the relationship between organizational learning and the absorptive capacity in organizations demonstrating theirrelationshipwith knowledgemanagement. For that, a theoretical research was carried out for exploratory-descriptive purposes by means of a bibliographical survey. The found theoretical elements confirmed the relevance of this research and they identified that knowledge is an input of the absorptive capacity and organizational learning, evidencing that knowledge management plays a key role in the integration between those two themes and that to amplify its analysis the interdisciplinary approach of the themes is imperative.
\end{abstract}

\section{REFERÊNCIAS}

ALVES, Karin Soares; STEIL, Andrea Valéria; SANTOS, Jane Lúcia Silva Santos. Processo de aprendizagem de grupo em uma empresa de tecnologia da informação. In: XV Semead: Seminários em Administração. Anais. 2012. Disponível em: <http://zip.net/bktypY>. Acesso em: 22 out. 2016.

ANGELONI, Maria Terezinha; STEIL, Andrea Valéria. Alinhamento de aprendizagem, estratégias e conhecimento organizacional. In: TARAPANOFF, Kira. (Org.) Aprendizado organizacional.

Curitiba: Ibpex, 2011.

AUSBURG, T. Becoming interdisciplinary: An introduction to interdisciplinary studies. New York: Kendall/Hunt Publishing, 2006.

CAMISÓN, C.; FORÉS, B. Knowledge absorptive capacity: new insights for its conceptualization and measurement. Journal of Business Research, v. 63, n. 7, p. 707-715, jul. 2010. Disponível em: <http:// zip.net/bjtybt>. Acesso em: 24 out. 2016.

CHENG, J. L. et al. From the Editors: Advancing interdisciplinary research in the field of international business: Prospects, issues and challenges. Journal of International Business Studies, v. 40, n. 7, p. 10701074, Sep. 2009.

COHEN, W. M.; LEVINTHAL, D. A. Absorptive capacity: a new perspective on learning and innovation. Administrative Science Quarterly, vol. 35, p. 128-152, 1990. Disponível em: <http://zip. net/bwtxLV>. Acesso em: 22 out. 2016.

CROSSAN, M. M.; APAYDIN, M. A multidimensional framework of organizational innovation: a systematic review of the literature. Journal of Management Studies, v. 47, n. 6, 2010. Disponível em: <https://goo.gl/Ufrj8h>. Acesso em: 23 out. 2016.

CNI. Desafios para a Indústria 4.0 no Brasil. Confederação Nacional da Indústria: Brasília: CNI, 2016.34p.

Keywords: Organizational learning. Absorptive capacity.

Knowledge management. Interdisciplinarity.ciplinarity.. 
DA SILVA, Sergio Luís. Gestão do conhecimento: uma revisão crítica orientada pela abordagem da criação do conhecimento. Ciência da Informação, Brasília, v. 33, n. 2, p. 143-151, maio/ago, 2004. Disponível em: <http://zip.net/bntymm >. Acesso em: Acesso em: 22 out. 2016.

DAVENPORT, Thomas H. PRUSAK, Laurence. Conhecimento empresarial: como as organizações gerenciam o seu capital intelectual. 3. ed. Rio de Janeiro, RJ: Campus, 1998.

DÁVILA, Guillermo Antonio. Relações entre práticas de gestão do conhecimento, capacidade absortiva e desempenho: evidências do sul do Brasil. 2016. 217 f. Tese (Doutorado em Engenharia e Gestão do Conhecimento). UFSC: Florianópolis, 2016. Disponível em: <http://btd.egc.ufsc. br/?p=2232> Acesso em: 22 out. 2016.

EASTERBY-SMITH, Mark; LYLES, Marjorie. Introduction: Watersheds of organizational learning and knowledge management. In: EASTERBYSMITH; LYLES, Marjorie (org). Handbook of Organizational Learning and Knowledge Management. Malden: Blackwell, p.1- 15, 2005.

FIOL, C. AND LYLES, M. Organizational learning. Academy of Management Review, v. 10, n. 4, p. 803813, 1985. Disponível em: <http://zip.net/bbtx8p>. Acesso em: 23 out. 2016.

FREIRE, Patricia de Sá. Engenharia da integração do capital intelectual nas organizações intensivas em conhecimento participantes de fusões e aquisições. 2012. 332 f. Tese (Doutorado em Engenharia e Gestão do Conhecimento). UFSC: Florianópolis, 2012. Disponível em: <http://zip.net/ bdtyNt>. Acesso em: 24 out. 2016.

FRIGOTTO, Gaudêncio. A interdisciplinaridade como necessidade e como problema nas ciências sociais. Ideação: revista do centro de educação e letras. Foz do Iguaçu, v.10, n. 01, p. 41-62, $1^{\circ}$ sem. 2008. Disponível em: <http://zip.net/bptyXB >. Acesso em: 23 out. 2016.
GARCÍA-MORALES, V. J.; RUIZ-MORENO, A.; LLORENS-MONTES, F. J. Effects of technology absorptive capacity and technology proactivity on organizational learning, innovation and performance: An empirical examination. Technology Analysis and Strategic Management, v. 19, n. 4, p. 527-558, 2007. Disponível em: <http://zip.net/bdtyNv>. Acesso em: 24 out. 2016.

HOTHO, J. J.; BECKER-RITTERSPACH, F.; SAKA-HELMHOUT, A. Enriching absorptive capacity through social interaction. British Journal of Management. v.23, n3, p. 383-401, Set. 2012. Disponível em: <http://zip.net/bjtybw $>$. Acesso em: 24 out. 2016.

JANSEN, J. P.; VAN DEN BOSCH, F. A. J.; VOLBERDA, H.W. Managing potencial and realized absorptive capacity: how do organizational antecedents matter. Academy of Management Journal, Barcelona: 2003. Disponível em: <http://zip. net/bhtymL >. Acesso em: 23 out. 2016.

KURTZ, Diego Jacob; SANTOS, Jane Lucia S.; STEIL, Andrea Valéria. Capacidade de Absorção do Conhecimento e Capacidades Dinâmicas no Contexto de Ambientes Turbulentos: uma análise da literatura. In: Encontro de Administração da Informação, IV, 2013, Bento Gonçalves. Anais. Bento Gonçalves: 2013. Disponível em: <http://zip.net/bntymp>. Acesso em: 21 out. 2016.

LANE, P.; KOKA, B.; PATHAK, S. The reification of absorptive capacity: a critical review and rejuvenation of the construct. Academy of Management Review, vol. 31, n. 4, p.833-863, 2006. Disponível em: <http:// zip.net/bwtxLZ>. Acesso em: 23 out. 2016.

LANE, P. J.; LUBATKIN, M. Relative absorptive capacity and interorganizational learning. Strategic Management Journal, v. 19, n.1, p. 461-477. 1998. Disponível em: <http://zip.net/bxtzfp >. Acesso em: 24 out. 2016.

LAURETH, Waleska Camargo. Convergência tecnológica, educação e trabalho: do discurso social global aos desafios regionais. Revista da ABET, v. 13, n. 2, p. 279-293, jul - dez. 2014. Disponível em: $<$ http://zip.net/bstyx1>. Acesso em 24 out. 2016. 
LEIS, Hector Ricardo. Sobre o conceito de interdisciplinaridade. Cadernos de Pesquisa Interdisciplinar em Ciências Humanas. Florianópolis, v. 06, n. 73, p. 01-23, ago. 2005. Disponível em: <http://zip.net/bmtx9P>. Acesso em: 23 out. 2016.

NONAKA, Ikujiro; TAKEUCHI, Hirotaka. Criação de conhecimento na empresa. Rio de Janeiro: Campus, 1997.

NORTH, K.; SILVA NETO, E.; DÁVILA, G. A. Vencendo os desafios do crescimento: o método "aprender a crescer" para pequenas e médias empresas brasileiras. Navus - Revista de Gestão e Tecnologia, v. 3, n. 1, p. 06-19, 2013. Disponível em: <http://zip.net/ bjtybC>. Acesso em: 24 out. 2016.

\section{ORGANIZAÇÃO PARA A COOPERAÇÃO E} DESENVOLVIMENTO ECONÔMICO - OCDE. Manual de Oslo: Diretrizes para coleta e interpretação de dados sobre inovação. 3.ed. Paris: OCDE, 2005.

Disponível em: <http://zip.net/brtyk1>. Acesso em: 23 out. 2016.

PICOLI, Florindo Rhaoni; TAKAHASHI, Adriana. Capacidade de Absorção, Aprendizagem Organizacional e Mecanismos de Integração Social. RAC, Rio de Janeiro, v. 20, n. 1, p. 1-20, Jan./Fev. 2016. Disponível em: <http://zip.net/btty1b>. Acesso em: 23 out. 2016.

POMBO, Olga. Interdisciplinaridade e integração dos saberes. Liinc em Revista, Rio de Janeiro, v.1, n.1, p. 3 -15 mar. 2005. Disponível em: <http://zip.net/ bvtyJq>. Acesso em: 22 out. 2016.

SANTOS, Jane Lucia Silva. Relações entre capacidade de absorção de conhecimento, sistemas de memória organizacional e desempenho financeiro. 2013. $234 \mathrm{f}$. Tese (Doutorado em Engenharia e Gestão do Conhecimento). UFSC: Florianópolis, 2013. Disponível em: <http://btd.egc. ufsc.br/?p=1657>. Acesso em: 24 out. 2016.

SERRAT, Olivier. Notions of Knowledge Management. Knowledge Solutions. 2008. Disponível em: <http://zip.net/bxtzfr >. Acesso em: 23 out. 2016. bltxMx>. Acesso em: 22 out. 2016.
SUN, P. Y. T.; ANDERSON, M. H. An examination of the relationship between absorptive capacity and organizational learning, and a proposed integration. International Journal of Management Reviews, v. 12, n. 2, p. 130-150, 2010. Disponível em: <http://zip. net/bjtybD>. Acesso em: 22 out. 2016.

\section{TEIXEIRA FILHO, Jayme. Gerenciando}

Conhecimento: como a empresa pode usar a memória organizacional e a inteligência competitiva no desenvolvimento de negócios. Rio de Janeiro: SENAC, 2000.

UENO, Alexandre Takeski; SANTOS, Neri dos; DANDOLINI, Gertrudes. Inteligência para competitividade: o papel do conhecimento na identificação de oportunidades de inovação. Revista Inteligência Conpetitiva. v.6, n. 2, p. 229-256, abr.jun. 2016. Disponível em: <http://zip.net/bytyKD>. Acesso em: 25 out. 2016.

UFSC. Universidade Federal de Santa Catarina. Programa de Pós-Graduação em Engenharia e Gestão do Conhecimento (PPGEGC). EDITAL 003/PPGEGC/2016: Instruções, Informações e Critérios Complementares. 2016. Disponível em: <http://zip.net/bgtygZ>. Acesso em: 01 dez. 2016.

VERSIANI, A. F.; FISCHER, A. L. (2008). A aprendizagem organizacional como um campo específico de conhecimento no cenário dos estudos organizacionais. Revista E\&G Economia e Gestão, v. 8, n. 18, 10-31. Disponível em: <http://zip.net/ bxtzft>. Acesso em: 22 out. 2016.

ZAHRA, S. A.; GEORGE, G. Absorptive capacity: A review, reconceptualization, and extension.

Academy of Management Review, v. 27, n. 2, p. 185203, Apr 2002. Disponível em: <http://zip.net/

ZANGISKI, Marlene Aparecida da Silva; LIMA, Edson Pinheiro de; COSTA, Sérgio Eduardo Gouveia da. Aprendizagem organizacional e desenvolvimento de competências: uma síntese a partir da gestão do conhecimento. Produto e Produção, v. 10, n. 1, p. 5474, fev. 2009. Disponível em: <http://zip.net/bltxMy>. Acesso em: 23 out. 2016.

Data de recebimento: 02/12/2016

Data de aprovação: 13/12/2016 


\section{SOBRE A AUTORA}

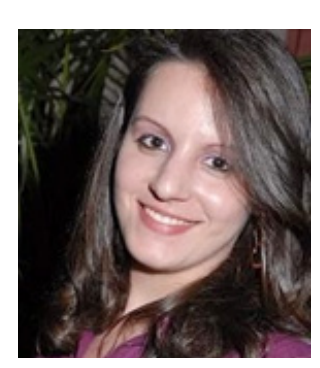

Regina Wundrack do

Amaral Aires

Especialista em Gestão

Empresarial pela

Universidade do Oeste de Santa Catarina (UNOESC),

Campus de São Miguel do

Oeste, e com MBA em Gestão Estratégica de Instituições de Educação Profissional e Tecnologia pela Faculdade de Tecnologia SENAI/SC Florianópolis. Bacharel em Secretariado Executivo graduada pelaUNOESC.Trabalha no SENAISanta Catarina desde 2007, sendo que até julho de 2013 atuou na Unidade de São Migueldo Oeste, desempenhando funções administrativas na área de qualidade, marketing, coordenação de cursos de qualificação profissional e docência nas áreas de qualidade e marketing. Nos anos de 2012 e 2013, foi coordenadora do Núcleo de Apoio Administrativo. Atualmente integra a equipe da Coordenadoria de Educação Profissional da Gerência de Educação e Tecnologia do Departamento Regional do SENAI Santa Catarina, junto à Modalidade de Cursos Técnicos como Especialista em Educação e é responsável pela coordenação do Programa Nacional de Acesso ao Ensino Técnico e Emprego - PRONATEC.

\section{***}

